\title{
A Note on Generated Systems of Sets*
}

\author{
ARLETE CERQUEIRA LIMA
}

\section{I - Introduction}

The existence of a smallest algebra ( $\sigma$-algebra) containing a given collection of subsets of a set $X$ is an elementary and well known fact (cf. [3] p. 22). Considering a modified definition of algebra ( $\sigma$-algebra) (see the definition below) where the unit is not necessary the whole $X$, the existence of the smallest algebra ( $\sigma$-algebra) in this generalized sense can not be guaranteed. This fact was noted by A. B. Brown and G. Freilich, in [1], where a necessary and sufficient condition was given as follows:

THEOREM. Let $\mathscr{S}$ be a collection of subsets of $X$ such that $\bigcup \mathscr{S} Z \neq X$. A necessary and sufficient condition for the existence of a smallest $\sigma$-algebra (in the sense of the below definition) containing $\mathscr{S}$. is the existence of a countable collection $\left\{S_{n}\right\}, S_{n} \in \mathscr{S}$ such that $Z=\bigcup_{n=1}^{\infty} S_{n}$.

Note that the condition $Z \neq X$ which is not stated in [1] can not be omitted.

The notations of algebra and $\sigma$-algebra are in the theory of quantum probability spaces frequently substituted by that of $q$-algebra and $q$ - $\sigma$-algebra. The last are usually named s-class or $\sigma$-class respectively (see [2], [4], [5]).

The difference between the notions $q$-algebra and $q$ - $\sigma$-algebra is in substituting the condition of closedness with respect to unions, by the condition of closedness wth respect to disjoint unions (see the definition below). When the $q$-algebras and $q$ - $\sigma$-algebras with a unit different of $X$ are considered, evidently the existence of a smallest one needs not be guaranteed. It seems to be natural that a necessary and sufficient condition for the existence can be obtained if the condition $Z=\bigcup_{n=1}^{\infty} S_{n}$ in Theorem is substituted by the *Recebido pela SBM em 2 de outubro de 1973. 
similar one where $\left\{S_{n}\right\}$ are required to be pairwise disjoint. But this is not the case as we show in this note.

\section{II - Notations and notions}

Definimion. A nonempty collection $\mathscr{A}$ of subsets of a set $X$ is said to be $a l$ gebra if

a) there exists $E \in \mathscr{A}$ such that $E \supset A$ for any $A \in \mathscr{A}$;

b) if $A \in \mathscr{A}$ then $E-A \in \mathscr{A}$;

c) if $A, B \in \mathscr{A}$ then $A \cup B \in \mathscr{A}$.

Note 1. $E$ is called the unit of $\mathscr{A}$. In the case $E=X$ we get the definition in the usual sense (see [3]).

Note 2. To obtain the notion of $\sigma$-algebra we substitute c) by the usual condition of countable unions.

Note 3. The notions of ring and $\sigma$-ring are used in the same sense as in [3].

Definition 2. A nonempty collection $\mathscr{A}$ of subsets of $X$ is said to be a q-ring if

a) $A, B \in \mathscr{A}, A \subset B$ implies $B-A \in \mathscr{A}$;

b) $A, B \in \mathscr{A}, A \cap B=\phi$ implies $A \cup B \in \mathscr{A}$.

If moreover there is $E \in \mathscr{A}$ such tht $A \subset E$ for any $A \in \mathscr{A}$ then is said to be a $q$-algebra.

Note 4. The notions of $q$ - $\sigma$-ring and $q-\sigma$-algebra are defined in a natural way substituting the conditions of the closedness with respect to finite disjoint unions by that countable disjoint unions in the $q$-anel and $q$-algebra respectively.

\section{III - Results}

THEOREM 1. Let $\mathscr{S}$ be a collections of subsets of $X$ such that $\bigcup \mathscr{S}=Z$. 
i) A sufficient condition for the existence of a smallest $q$-algebra ( $q$ - $\sigma$-algebra) containing $\mathscr{S}$ is

$$
\begin{gathered}
Z=\bigcup_{i=1}^{n} S_{i} \quad \text { where } \quad S_{i} \in \mathscr{P}, i=1,2, \ldots n, S_{i} \cap S_{j}=\phi . \quad \text { if } \quad i \neq j \\
\left(Z=\bigcup_{i=1}^{\infty} S_{i}, S_{i} \in \mathscr{S}, S_{i} \cap S_{j}=\phi, i, j=i, 2, \ldots, \quad \text { if } \quad i \neq j\right)
\end{gathered}
$$

ii) If $Z \neq X$ a necessary condition for the existence of a smallest q-algebra (q- $\sigma$-algebra) containing $\mathscr{S}$ is

$$
\begin{aligned}
& Z=\bigcup_{i=1}^{n} S_{i}, \quad S_{i} \in \mathscr{S}, i=1, \ldots n . \\
& \left.Z=\bigcup_{i=1}^{\infty}, \quad S_{i} \in \mathscr{S}, i=1,2 \ldots\right) .
\end{aligned}
$$

iii) The condition i) is not necessary and the condition ii) is not sufficient.

PRoof. We shall give a proof for $q$-algebras (the proof for $q-\sigma$-algebras is analogous.

i) Considering all the $q$-algebras with the fixed unit $Z$ the usual approach gives the existence of the smallest one containing $\mathscr{S}$ belonging to this collection. Let $\mathscr{A}$ be this $q$-algebra. We shall prove that $\mathscr{A}$ is the smallest among all $q$-algebras containing $\mathscr{S}$, with or not the fixed unit $Z$. In fact, let $\mathscr{A}^{\prime} \supset \mathscr{S}$ be any $q$-algebra. The condition

$$
Z=\bigcup_{i=1}^{n} S_{i}, \quad S_{i} \in \mathscr{S}, \quad S_{i} \cap S_{j}=\phi \quad \text { if } \quad i \neq j .
$$

gives $Z \in \mathscr{A}^{\prime}$. Let $\mathscr{E}=\left\{A: A \in \mathscr{A}, A \in \mathscr{A}^{\prime}, Z-A \in \mathscr{A}^{\prime}\right\}$. The system $\mathscr{E}$ is a $q$-algebra with the unit $Z$ because $A_{1}, A_{2} \in \mathscr{E}, A_{1} \cap A_{2}=\phi$ implies $A_{1} \cup A_{2}$ $\in \mathscr{A}, A_{1} \cup A_{2} \in \mathscr{A}^{\prime}$ and $Z-\left(A_{1} \cup A_{2}\right)=\left(Z-A_{1}\right)-A_{2} \in \mathscr{A}^{\prime}$ because of the fact $A_{2} \subset Z-A_{1}$.

On the other hand $\mathscr{S} \subset \mathscr{E}$. Thus $\mathscr{A} \subset \mathscr{E}$. But $\mathscr{E} \subset \mathscr{A}^{\prime}$ hence, $\mathscr{A} \subset \mathscr{A}^{\prime}$.

ii) If $Z$ is not a finite union of the elements of $\mathscr{S}$ then it is possible using the method of [1] to construct an algebra $\mathscr{A}$ such that $\mathscr{A} \supset \mathscr{S}$ and $Z \notin \mathscr{A}$. A smallest $q$-algebra $\mathscr{A}^{\prime}$ containing $\mathscr{S}$ cannot exist. In fact, if it exists then it 
is easy to prove that $Z \in \mathscr{A}^{\prime}$. But $\mathscr{A}$ being algebra is also a $q$-algebra, hence $\mathscr{A}^{\prime} \subset \mathscr{A}$. Thus $Z \in \mathscr{A}$, what is a contraction.

iii) Let $X=\{1,2,3, \ldots, 8,9\}$ and $Z=\{1,2, \ldots, 8\}$.

Let $\mathscr{S}$ be the collection containing the set $\{1,2,3,4\}$ and all three elements subsets of $\{1,2, \ldots 8\}$. Evidently $Z$ is not a disjoint union of the elements of $\mathscr{S}$. Nevertheless the smallest $q$-algebra containing $\mathscr{S}$ exists and it is the $q$-algebra of all subsets of $Z$. Hence the condition i) is not necessary.

To show that (ii) is not sufficient let

$$
X=\{a, b, c, d\}, \quad \mathscr{S}=\{\{a, b\},\{b, c\}\} \quad \text { and } \cdot Z=\{a, b, c\} .
$$

It is easy to see that $\mathscr{A}=\{\{a, b\},\{b, c\},\{a, b, c\},\{c\},\{a\},\{a, c\},\{b\}, \phi\}$ is a $q$-algebra containing $\mathscr{S}$ with unit $E=\{a, b, c\}$

Since $\mathscr{A}$ is the smallest $q$-algebra of subsets of $Z$ with the unit $Z$ containing $\mathscr{S}$ then, as we know, if there exists a smallest $q$-algebra $\mathscr{A}^{*}$ containing $\mathscr{S}$ it should coincide with $\mathscr{A}$. But $\mathscr{A}$ is not the smallest $q$-algebra containig $\mathscr{S}$. In fact if

$$
\mathscr{B}=\{\{a, b\},\{b, c\},\{a, b, c, d\},\{c, d\},\{a, d\}, \phi\}
$$

then $\mathscr{B}$ is a $q$-algebra which contains $\mathscr{S}$, but $\mathscr{A} \not \mathscr{B}$.

Nore. The part (iii) of the preceeding theorem shows that an analogy of the Theorem proved in [1] is not valid for $q$-algebra $(q-\sigma$-algebras). The following is a necessary and sufficient condition for the existence of a smallest $q$-algebra and can be formulated in the same manner also for algebras.

THEOREM 2. Let $\mathscr{S}$ be a collection of subsets of $X$ such that $\bigcup \mathscr{I}=Z \neq X$. Denote by $\mathscr{A}_{Z}$ the smallest q-algebra of subsets of $Z$ which contains $\mathscr{I}$ and by $\mathscr{A}$ the smallest q-ring (which alwavs exists) containing $\mathscr{S}$. A necessary and sufficient condition for the existence of the smallest q-algebra $\mathscr{A}_{0}$ containing $\mathscr{S}$ is $\mathscr{A}=\mathscr{A}_{Z}$.

Proof. Let $\mathscr{A} \neq \mathscr{A}_{Z}$. Then evidently $Z \notin \mathscr{A}$. Choose $\alpha \in X, \alpha \notin Z$ and put

$$
\mathscr{B}=\{A: A \in \mathscr{A} \quad \text { or } \quad(Z \cup\{\alpha\})-A \in \mathscr{A}\} \text {. }
$$


$\mathscr{B}$ is a q-algebra which contains $\mathscr{S}$. The fact $\mathscr{S} \subset \mathscr{B}$ is obvious.

Now let $A, B \in \mathscr{B}, A \subset B$. If both $A, B \in \mathscr{A}, A \cap B=\phi$, then $A \cup B \in \mathscr{A}$, hence $A \cup B \in \mathscr{B}$. If under the same conditions $A \in \mathscr{A}$ and $Z \cup\{\alpha\}-B \in \mathscr{A}$, then $(Z \cup\{\alpha\})-(A \cup B)=(Z \cup\{\alpha\}-B)-A \in \mathscr{A}$. The case $B \in \mathscr{A},(Z \cup\{\alpha\})-$ $A \in \mathscr{A}$ is analogous. The case $(Z \cup\{a\})-A \in \mathscr{A},(Z \cup\{\alpha\})-B \in \mathscr{A}$ is not possible because $A, B$ are disjoint.

The fact that $A \subset(Z \cup\{\alpha\})$ for any $A \in \mathscr{B}$ is evident as well fact when $A \in \mathscr{B}$ also the complement $(z \cup\{\alpha\})-A \in \mathscr{B}$. Hence $\mathscr{B}$ is a $q$-algebra with the unit $Z \cup\{\alpha\}$.

Evidently $\mathscr{S} \subset \mathscr{B}$. But $Z \notin \mathscr{B}$ because $Z \in \mathscr{A}$ and $(Z \cup\{\alpha\})-Z=\{\alpha\} \notin \mathscr{A}$. Hence a smallest $q$-algebra $\mathscr{A}_{0}$, containing $\mathscr{S}$ doesn't exist Suppose it exists; then we have $Z \in \mathscr{A}_{0} \subset \mathscr{B}$, which is impossible.

Now let $\mathscr{A}=\mathscr{A}_{Z}$. Then $\mathscr{A}$ is a $q$-algebra which contains $\mathscr{S}$ with a unit $Z$. If $\tilde{A}$ is any $q$-algebra containing $\mathscr{S}$ then $\mathscr{A}$ is a $q$-ring. This $\mathscr{A} \supset \mathscr{A}=\mathscr{A}_{Z}$. Hence $\mathscr{A}_{0}=\mathscr{A}_{Z}=\mathscr{A}$ is the smallest $q$-algebra which contains $\mathscr{S}$.

\section{BIBLIOGRAPHY}

[1] A. B. Brown and G. FreIlich, $A$ condition for existence of a smallest Borel algebra containing a given collection of sets, Enseignment Math. 2 (1967), 107-109.

[2] GudDER, Quantum probability spaces, Proceedings of the American Mathematical Society 21 (1969) pp. 296-302.

[3] P. Halmos, Mesure Theory, D. Van Nostrand Company, Inc.

[4] Neubrunn, A note on quantum probability spaces, Proceedings of the American Mathematical Society vol. 25, n. ${ }^{\circ} 3$, July, 1970.

[5] Neubrunn, $A$ certain generalized randon variables (to appear). 